\title{
Innate immune cell-epithelial crosstalk during wound repair
}

\author{
Jennifer C. Brazil, Miguel Quiros, Asma Nusrat, and Charles A. Parkos \\ Department of Pathology, University of Michigan, Ann Arbor, Michigan, USA.
}

\begin{abstract}
Skin and intestinal epithelial barriers play a pivotal role in protecting underlying tissues from harsh external environments. The protective role of these epithelia is, in part, dependent on a remarkable capacity to restore barrier function and tissue homeostasis after injury. In response to damage, epithelial wounds repair by a series of events that integrate epithelial responses with those of resident and infiltrating immune cells including neutrophils and monocytes/macrophages. Compromise of this complex interplay predisposes to development of chronic nonhealing wounds, contributing to morbidity and mortality of many diseases. Improved understanding of crosstalk between epithelial and immune cells during wound repair is necessary for development of better pro-resolving strategies to treat debilitating complications of disorders ranging from inflammatory bowel disease to diabetes. In this Review we focus on epithelial and innate immune cell interactions that mediate wound healing and restoration of tissue homeostasis in the skin and intestine.
\end{abstract}

\section{Introduction}

Epithelial barriers at mucosal and dermal surfaces form a protective shield against microbial invasion and environmental damage. Perpetual epithelial renewal is facilitated by stem and progenitor cells that balance proliferation and differentiation signals to continuously replace terminally differentiated or dying cells. Rapid self-renewal also supports epithelial cells' essential role in barrier regulation and wound repair. Wound healing is a complex process characterized by four overlapping stages: hemostasis, inflammation, proliferation/re-epithelization, and remodeling. Dysregulation of any stage is linked to an increased risk of developing chronic nonhealing wounds, representing a substantial worldwide health care burden associated with considerable morbidity and mortality $(1,2)$.

During normal gut function, the mucosal epithelium is repetitively injured through mechanical and chemical interactions with luminal contents. Mucosal injuries are constantly repaired to maintain gut homeostasis and provide sufficient nutrients while simultaneously preserving barrier function. Typically, superficial mucosal damage is associated with acute intestinal inflammation that resolves quickly without substantial fibrosis or compromised gastrointestinal function. However, chronic disorders of the digestive tract such as inflammatory bowel disease (IBD; encompassing Crohn's disease and ulcerative colitis) are characterized by recurring mucosal inflammation and injury (reviewed in ref. 3). While IBD etiology remains elusive, its pathobiology is closely linked to dysregulated intestinal barrier function and insufficient healing, which is associated with perturbed mucosal homeostasis (4). Approximately 3 million individuals in the United States suffer from IBD (1).

Authorship note: JCB and MQ contributed equally to this work. Conflict of interest: The authors have declared that no conflict of interest exists. Copyright: (c) 2019, American Society for Clinical Investigation. Reference information: J Clin Invest. 2019;129(8):2983-2993. https://doi.org/10.1172/JCl124618.
Like mucosal wound repair in the gut, superficial epidermal injuries of the skin such as first-degree burns do not undergo major remodeling during healing and usually do not produce scarring. However, deeper transdermal injuries heal with considerable remodeling, often resulting in fibrosis, permanent scarring, and loss of skin appendages including hair follicles and sebaceous glands. Failure to resolve cutaneous wounds, formation of chronic ulcers, and excessive scarring represent appreciable health and economic burdens to individuals with a number of conditions, including vascular insufficiency caused by factors such as aging, diabetes mellitus, and smoking (5).

Given the devastating impact of defective intestinal and dermal wound healing on human health, this Review highlights current mechanisms regulating epithelial wound repair, focusing on the intestine as a well-studied example of a simple columnar epithelium and the skin as an example of a more complicated stratified epithelium. As other Reviews in this JCI series discuss adaptive immune responses, we limit discussion to the role of epithelial and innate immune cell interactions in wound healing. We discuss roles of epithelial cells, neutrophils, monocytes, and macrophages in wound repair and address interactions between these cell types.

\section{Epithelial cells in cutaneous and intestinal wound repair}

Intestinal epithelium. The intestinal epithelium lines the largest mucosal surface in the body and provides critical barrier between microbiota and mucosal immune cells. The initial response to intestinal epithelial injury involves hemostasis, which limits blood loss and seals damaged tissue. With the onset of hemostasis, the inflammatory response begins and includes critical contributions from epithelial and immune cells. In vitro and in vivo studies of human, rabbit, and rodent epithelia reveal that within minutes of intestinal mucosal injury, epithelial cells within crypts adjacent to the wound begin migrating as a collective sheet to cover injured/ denuded surfaces $(6-10)$. During repair, epithelial cells under- 


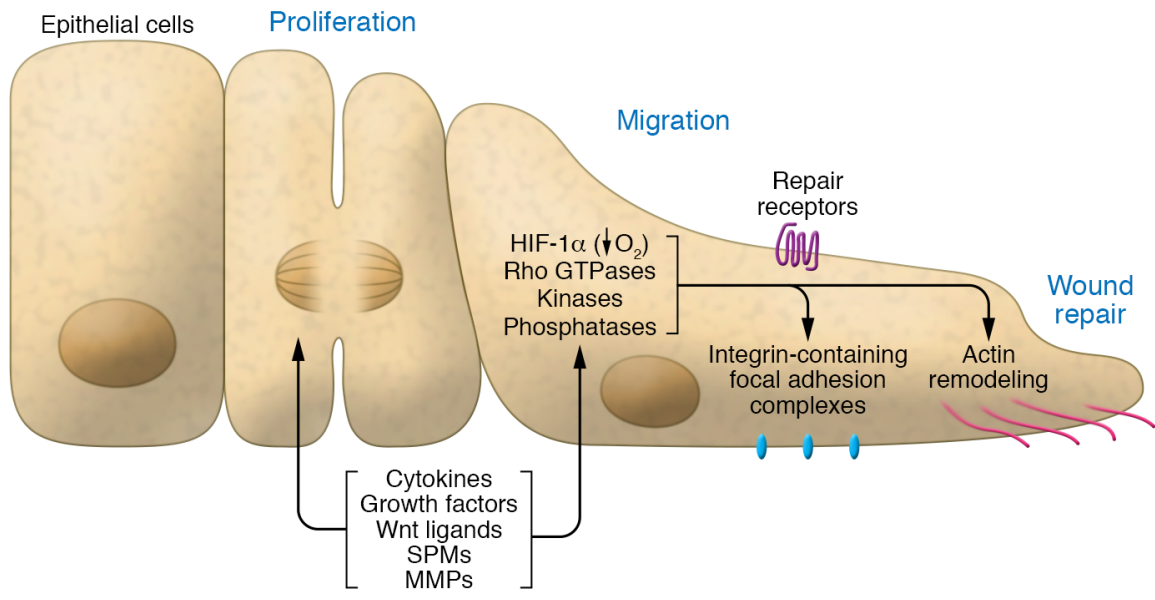

Figure 1. Epithelial reparative triggers and

events. Cytokines, growth factors, Wnt ligands, SPMs, and MMPs released in the wound microenvironment in response to injury support epithelial cell proliferation as well as migration. Dynamic remodeling of focal adhesion complexes and actin promotes interactions with the ECM that facilitate the epithelial sheet's migration. Following initial epithelial cell migration, keratinocytes peripheral to the leading edge proliferate and mature to restore epithelial barrier homeostasis and function.

go morphologic changes in shape, modify cell-cell contacts, and migrate collectively to reseal the barrier.

Collective epithelial cell migration during wound healing requires cytoskeletal remodeling and active crosstalk between cell matrix and cell-cell junction proteins. To facilitate migration, integrin-containing focal adhesive complexes are dynamically remodeled in concert with intracellular F-actin-rich extrusions at the leading edge that adhere to the extracellular matrix (ECM) to propel epithelial sheet migration (Figure 1 and refs. 11, 12).

Dermal epithelium. In contrast to the single layer of columnar epithelial cells lining the gut, a multilayered squamous epithelium lines the skin. Dermal epithelial cells form an important physical barrier against the environment, protecting against pathogens, xenobiotics, and dehydration $(13,14)$. Like intestinal epithelium, a reservoir of dynamic basal stem cells capable of generating all skin cell lineages facilitates ongoing cutaneous tissue turnover and skin regeneration $(15,16)$. The outermost epidermal layer comprises multiple layers of flattened dead cells (stratum corneum), making skin highly impermeable. However, skin epidermis interfaces with the outside world, making it particularly prone to injury, necessitating frequent repair.

Like repair of mucosal wounds, repair of skin injury depends on activation of the coagulation cascade followed by immune cell infiltration of wounds, contributing to protection against invading pathogens and epithelial repair (17). As in the intestine, skin reepithelization also involves collective migration of keratinocytes across the injured dermis. Following initial epithelial cell migration, keratinocytes behind the leading edge proliferate and mature to restore epithelial barrier function. Using whole-mount epidermis, Aragona et al. confirmed the existence of leading-edge, nonproliferative migrating cells and a proliferative hub of stem cells and their progeny (16), highlighting molecular signatures associated with these two distinct epidermal compartments. Upon reepithelization, new highly vascularized connective tissue containing fibroblasts, granulocytes, macrophages, and loosely organized extracellular collagen is deposited into the wound bed. The final stage of skin wound repair involves tissue remodeling that begins 2 to 3 weeks following initial injury and lasts up to a year or more, depending on wound severity (18).

Epithelial repair signaling. During wound remodeling in the skin and gut, matrix metalloproteinases (MMPs) cleave or degrade ECM components. Humans express 24 MMPs that regulate diverse activities important for ECM remodeling and forward movement of the epithelium (reviewed in ref. 19). MMP endoproteinase activity facilitates removal of disorganized structural proteins from healing wounds to make room for newly synthesized collagen. Furthermore, MMP-mediated conversion of type III collagen to more stable type I collagen increases wound tensile strength. Fibroblast- and keratinocyte-derived MMP-1 promotes breakdown of excess collagen in murine and rabbit models of skin repair (20-22). Though not expressed in skin, epithelial cellderived matrilysin (MMP-7) is reportedly the key MMP involved in repairing injured intestinal mucosa in humans $(23,24)$.

Signals that trigger epithelial migration and proliferation from injured sites are incompletely understood. Loss or modification in cell-cell contact and release of intracellular molecules initiates repair (25). These events set the stage for recruiting leukocytes and mesenchymal cells that orchestrate wound repair. Formylated peptides and ATP released by damaged cells, also referred to as damage-associated molecular patterns (DAMPs), orchestrate repair by promoting epithelial cell migration and proliferation. Epithelial wounds are also a source of intracellular $\mathrm{Ca}^{++}$waves that are rapidly transmitted into surrounding tissues to influence repair. Furthermore, ROS signaling and wound-associated physical cues influence epithelial repair. Small GTPases in the Rho family regulate remodeling of $\mathrm{F}$-actin, intercellular junctions, and cell-matrix adhesions (26) and are crucial for epithelial cell migration and wound sealing. Similarly, the Rho GTPase Rac1 promotes intestinal epithelial proliferation by targeting $\beta_{1}$-integrin in cellular protrusions and modulating actin dynamics (26).

Reparative signaling events are also regulated by extracellular mediators in the epithelial milieu, including annexin A1, annexin A2, and serum amyloid A1, which have been shown to influence integrin localization, focal adhesion kinase activation, and cell matrix remodeling in mouse and human intestinal mucosa (27-30). After injury, chemokines/cytokines and growth factors play crucial roles in epithelial cell adhesion, migration, proliferation, and differentiation. TGF- $\beta$-dependent signaling pathways mediate the regulatory effects of many repair mediators, including PDGF, EGF, VEGF, IL-1, IL-2, IL-6, and IFN- $\gamma$ (6). Canonical and noncanonical Wnt proteins also modulate epithelial wound repair. A recent in vivo study revealed a role of Wnt5a in orchestrating colonic crypt 
regeneration via TGF- $\beta$ signaling (31). In addition, while traditionally considered a proinflammatory cytokine, recent evidence demonstrated that TNF- $\alpha$ promotes mucosal wound repair in mice by activating Wnt/ $\beta$-catenin signaling, increasing epithelial cell proliferation, and upregulating expression of receptors that promote intestinal healing (Figure 1 and refs. 32, 33).

In summary, intestinal and cutaneous wound repair is in part facilitated by remarkable migratory and proliferative capabilities of epithelial cells. In the following sections, we highlight the complex spatial and temporal interplay between wound-associated neutrophils, monocytes, and macrophages as well as the crosstalk between these innate immune cells and dermal and intestinal epithelial cells during tissue repair.

\section{Innate immune cells in intestinal and dermal repair}

Neutrophils. Neutrophils are the first immune cells to infiltrate wounded tissues, arriving in large numbers in response to DAMPs released from injured and necrotic cells. Murine neutrophil recruitment to wounded tissues begins 4 to 6 hours after initial injury, with maximum numbers detected after 18 to 24 hours (34, 35). The neutrophil's role in wound healing can be viewed as a double-edged sword (36). Too few neutrophils risks infection and delayed healing (37), whereas overpersistence of neutrophils in injured tissues also delays healing through collateral tissue damage. For example, neutrophils contribute to the crypt loss and ulceration that are pathological hallmarks of ulcerative colitis, and excessive neutrophil infiltration parallels disease severity and patient symptoms (38-40). Therefore, neutrophil activation and migration in response to dermal or mucosal injury is tightly regulated. Impaired leukocyte trafficking delays cutaneous wound healing in mice $(41,42)$, highlighting neutrophils' critical importance in orchestrating efficient wound repair. Similarly, neutrophil depletion in damaged intestinal mucosa was associated with increased inflammation, impaired intestinal mucosal repair, and slower recovery from colitis in vivo $(43,44)$. Furthermore, individuals with neutropenia (or deficiencies in neutrophil trafficking or function) display not only higher risk for developing wound infections but also impaired wound healing $(37,45,46)$.

While many previous studies focused on neutrophil trafficking (reviewed elsewhere in refs. 47, 48), the DAMP-triggered mechanisms that facilitate neutrophil migration into skin and intestinal wounds are not yet well described. Once recruited to wounded dermal or intestinal tissues, neutrophils prevent infection by eradicating microbes that enter through disrupted epithelial barriers. Neutrophils destroy invading microbes through phagocytosis, or sometimes NETosis (formation of extracellular traps; ref. 49), while releasing antimicrobial peptides (AMPs, including cathelicidins and $\beta$-defensins), ROS, and cytotoxic enzymes such as elastase and myeloperoxidase (Figure 2 and ref. 50). To produce microbicidal ROS, neutrophils consume large amounts of oxygen, generating a hypoxic microenvironment within wounded tissues that results in stabilization of the transcription factor HIF- $1 \alpha$ in human and murine intestinal mucosa $(51,52)$. In wounded intestinal mucosa, HIF-1 $\alpha$ stabilization results in enhanced epithelial expression of intestinal trefoil factor (ITF). ITF activates epithelial MAPK signaling and induces reorganization of cell-cell junction proteins including E-cadherin, promoting epithelial mobility and barrier restitution following injury in vitro and in vivo $(52,53)$. HIF-1 $\alpha$ also promotes transcriptional upregulation of genes that enhance cutaneous wound repair, including metabolic proteins, adhesion proteins, soluble growth factors (TGF- $\beta$ and VEGF), and ECM components $(54,55)$. Therefore, neutrophil-mediated HIF- $1 \alpha$ stabilization in wound microenvironments acts through epithelial cells to promote barrier restitution and a faster return to tissue homeostasis.

In addition to eliminating microbes and modulating the wound microenvironment through oxygen metabolism, neutrophils release pro-repair cytokines, chemokines, and growth factors that signal through wound-associated immune and epithelial cells to promote healing. Following mucosal damage, infiltrating neutrophils secrete TGF- $\beta$ to activate MEK1/2 signaling and induce intestinal epithelial cell-mediated production of the EGF-like molecule amphiregulin (AREG) (56). AREG promotes intestinal epithelial cell differentiation and proliferation in a positive manner to facilitate efficient return to mucosal homeostasis in vivo $(56,57)$. TGF- $\beta$ also accelerates re-epithelization, angiogenesis, and granulation tissue formation in healing murine and human skin wounds (58-60). However, unlike healing intestinal mucosa, neutrophils in skin wounds are not yet identified as an important source of TGF- $\beta$. While not implicated in TGF- $\beta$ production, human neutrophils that migrate into skin wounds upregulate a transcriptional program that includes chemoattractants (e.g., CCL-2 and MIP1 $\alpha$, also known as CCL-3) and genes that promote angiogenesis (VEGF, IL-8, GRO- $\gamma$, and CCL-2), proliferation, and activation of keratinocytes and fibroblasts (IL-8, IL-1 $\beta$, and CCL-2) (61-63). Moreover, several studies reported that neutrophils are an important source of de novo TNF- $\alpha$ synthesis in healing mouse skin lesions $(64,65)$. While TNF- $\alpha$ is traditionally considered a proinflammatory mediator, it also mediates crucial pro-repair mechanisms, including stimulation of fibroblast proliferation, re-epithelization, and angiogenesis (66).

Neutrophils recruited to wounds also respond to the proinflammatory cytokine-rich milieu by producing CC chemokines such as CCL-20 (67), which attracts CCR-6-expressing inflammatory monocytes into murine injured skin (68). Recent work identified tissue-infiltrating neutrophils as a major source of IL-23 in the intestines of individuals with IBD (69). Furthermore, upon stimulation with IL-23 and TNF- $\alpha$, murine and human colonic neutrophils produce IL-22, a member of the IL-10 superfamily of cytokines. In murine intestinal wounds, neutrophil-produced IL-22 stimulated intestinal epithelial production of AMPs RegIII $\beta$ and S100A8 and increased epithelial proliferation, differentiation, and migration (70-72). Intestinal injury induces another IL-1 family member, IL-36, in epithelial cells and macrophages, and signaling through IL-36R promotes neutrophil recruitment, IL-22 production, and murine intestinal epithelial repair (73). In murine skin, it is known that IL-22 mediates interactions between immune cells and fibroblasts to promote wound healing $(74,75)$. However, murine neutrophils have not yet been identified as a prominent source of IL-22 during skin repair.

An additional mechanism whereby neutrophil-epithelial crosstalk promotes mucosal wound healing is via production of chemical mediators, including diadenosine triphosphate (Ap3A). 




Figure 2. Proinflammatory stage of wound healing. Neutrophils are the first responders to epithelial injury. They clear bacteria present at the wound site, limiting infection, and secrete proinflammatory TNF- $\alpha$, which stimulates fibroblast proliferation and angiogenesis.

Human colonic epithelial cells metabolize neutrophil-produced Ap3A to adenosine, resulting in downstream activation of surface adenosine receptors and enhanced epithelial barrier function and mucosal wound-healing responses in the gut $(76,77)$. In the mucosa, neutrophil-derived adenosine signals primarily through cAMP (78), which increases expression of human epithelial tight junction proteins including ZO-1 and occludin and modulates actin and intermediate filament dynamics (79). Like its pro-repair effects in the intestine, topical application of adenosine promotes cutaneous wound healing by stimulating angiogenesis and suppressing inflammatory cell function in mice (80). However, no direct effects of neutrophil-derived adenosine on skin epithelia have been reported to date. Taken together, these studies highlight the range of neutrophil-produced mediators acting on epithelial cells and wound-associated immune cells to promote cutaneous and intestinal wound healing.

The above evidence highlights the dual roles of woundassociated neutrophils that were once thought to simply maintain sterility following injury to include crucial immunomodulatory and pro-resolving or wound-healing functions. Further development of sophisticated imaging methodology including intravital microscopy (81) combined with transgenic strategies that specifically target/label neutrophil subsets in vivo (82) will allow detailed mechanistic analyses of neutrophil behavior within wound-healing environments. Furthermore, recent advances in understanding neutrophil plasticity and identifying distinct neutrophil subsets (83) should be exploited to develop therapeutic strategies to selectively deplete inflammatory neutrophil populations from poorly healing cutaneous and intestinal wounds. Additional studies are needed to identify markers and develop antibodies and small-molecule inhibitors that specifically target inflammatory neutrophils in wounds to promote repair and reduce chronic tissue damage in the skin and gut.

Monocytes. Following the initial neutrophil wound influx, epithelial, endothelial, lamina propria, and infiltrating immune cells release chemokines including CCL-20 and CCL-2 (84). These mediators facilitate subsequent recruitment of circulating monocytes into sites of tissue damage (Figure 2 and ref. 85). Wound-infiltrated monocytes play crucial roles in orchestrating tissue repair, including regulating angiogenesis, clearing cellular debris, and recruiting additional immune cells. Monocytes recruited into wounded tissues further differentiate into macrophages and/or DCs. In murine wounds, chemokines including CCL-2 and CX3CL-1 and their respective receptors, CCR-2 and CX3CR-1, regulate monocyte recruitment. Previous studies show that CX3CR-1 and CCR-2 are essential for wound repair in vivo: Cx3cr-1-null mice have delayed healing in skin wounds, and inhibiting CX3CR-1 signaling decreases skin angiogenesis and wound repair (86). In the gut, Ly6 $\mathrm{C}^{\text {hi }}$ monocyte recruitment requires CCR-2, and CCR-2-deficient mice have reduced numbers of monocyte-derived macrophages in wounds (87). Other ligands/ receptors involved in monocyte trafficking include CCR-1/CCL-3, CCR-5/CCL-5, CCR-6/CCL-20, CCR-7/CCL-19, and CCR-8/ CCL-1 (reviewed in ref. 88). 


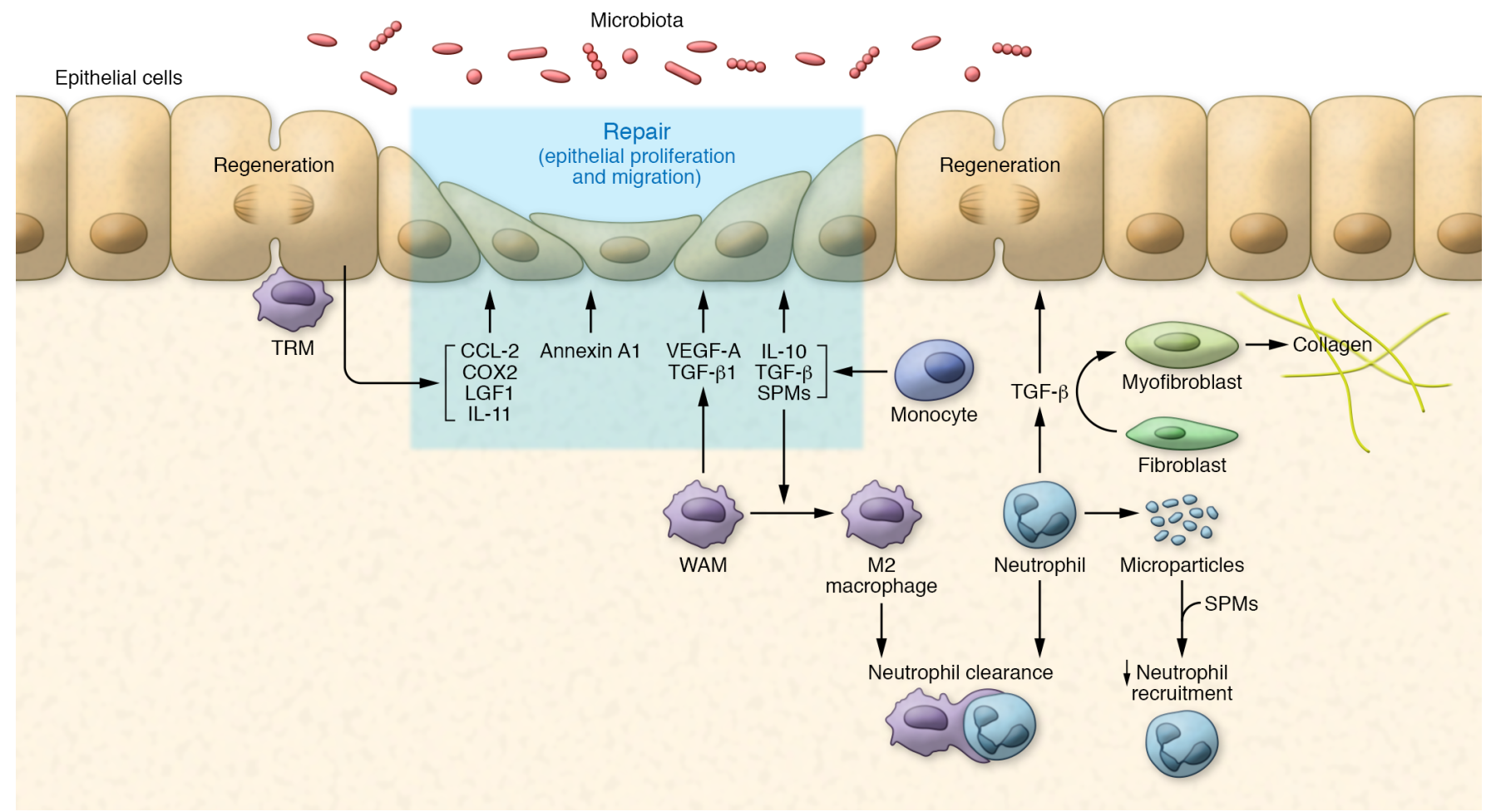

Figure 3. Resolution of inflammation and repair. Regenerating epithelial cells express pro-repair molecules including CCL-2, COX2, LGF1, and IL-11, possibly as a result of their activation by TRMs. Wound-associated macrophages (WAMs) and neutrophils also produce pro-repair signals, including annexin A1, VEGF-A, TCF- $\beta$, IL-10, and SPMs, that enhance resolution of inflammation at the wound site. In addition to supporting epithelial repair and migration, these pro-repair signals polarize macrophages to M2-like phenotypes that clear apoptotic neutrophils. In the presence of SPMs, neutrophil-derived microparticles may serve as a negative feedback mechanism to suppress additional neutrophil recruitment. TCF- $\beta$ also stimulates fibroblast differentiation into myofibroblasts, which produce collagen that provides structural support to the healing epithelium.

Interestingly, in mice, some monocytes that leave the circulation to migrate into injured tissues do not differentiate into tissue macrophages or DCs but instead undergo apoptosis and are removed from wound sites. Before removal, these monocytes contribute to wound-healing responses by releasing cytokines and chemokines (88). Tissue-infiltrating monocytes can have inflammatory or antiinflammatory/pro-repair properties. Inflammatory monocytes, typically characterized as $\mathrm{Gr} 1^{+} \mathrm{Ly} 6 \mathrm{C}^{\mathrm{hi}}$, CCR $-2^{+}$, $\mathrm{CX} 3 \mathrm{CR}-1^{10}$ in mice or $\mathrm{CD}^{+} 4^{+}, \mathrm{CD} 16^{-}$in humans, are the major population of mononuclear cells initially recruited to sites of injury (Figure 3). They are a potent source of proinflammatory cytokines such as IL- 6 and TNF- $\alpha$. Shortly after arrival of inflammatory monocytes into wound sites, monocytes with antiinflammatory properties marked by expression of $\mathrm{Gr}^{-}{ }^{-} \mathrm{Ly} 6 \mathrm{C}^{\mathrm{lo}}, \mathrm{CCR}-2^{-}, \mathrm{CX} 3 \mathrm{CR}-1^{\text {hi }}$ in mice and $\mathrm{CD} 14^{\mathrm{lo}}, \mathrm{CD} 16^{+}$in humans are observed. Antiinflammatory monocytes release pro-repair molecules such as VEGF and IL-10, promoting cell proliferation and angiogenesis (Figure 2 and ref. 88). While precise mechanisms are unclear, differential chemoattractant signaling from CCR-2 versus CX3CR-1 may regulate recruitment of proinflammatory versus pro-repair monocytes into murine healing wounds (89). However, it remains unclear whether infiltrated monocytes transition from a proinflammatory to an antiinflammatory phenotype before differentiating into macrophages, or whether independent monocyte populations migrate from the blood and differentiate into these cell types.
Both healthy intestine and skin contain resident monocytederived macrophages (90). Given that skin and intestinal epithelia are constantly exposed to microorganisms and their products, it follows that there is a dynamically changing population of associated macrophages. Continuous exposure to commensal microorganisms may be viewed as a stimulus that maintains "low-grade" chronic inflammation and induces monocyte recruitment (91). In summary, monocytes migrate to sites of injury (92) and secrete soluble mediators that contribute to wound repair. While many studies focus on macrophage functions in wound healing, the importance of infiltrating monocytes in mediating key aspects of skin and intestinal wound repair remains understudied.

Macrophages. Macrophages contribute to wound repair and tissue remodeling by clearing apoptotic neutrophils (efferocytosis) and helping to reduce autoimmune and chronic inflammatory responses (92). These effector functions are achieved, in part, through secretion of cytokines, growth factors, and specialized pro-resolving mediators (SPMs) (93). Wound-associated macrophages undergo polarization, a process involving integration of complex signals from the microenvironment followed by commitment to a functional program directed at restoring tissue homeostasis. Polarization continuously changes throughout the phases of wound healing. Historically, macrophage characterization was based on M1 (inflammatory) or M2 (antiinflammatory/prorepair) phenotypes. M1 macrophages are induced by inflammatory 
stimuli such as lipopolysaccharide and, when stimulated, release proinflammatory cytokines such as TNF- $\alpha$, IL-1 $\beta$, IL-6, IL-8, and IL-12. In contrast, M2 macrophages are induced by IL-4/IL-13 as well as IL-10. M2 macrophages release antiinflammatory/prorepair molecules including TGF- $\beta$, IL-10, and SPMs such as maresins, resolvins, and protectins (see ref. 94 for a detailed review on SPMs, and refs. 95, 96). It is increasingly appreciated that M1/M2 macrophage classification is oversimplified; substantial overlap in the responses of these two types of macrophages is undoubtedly due to intermediate or transitional stages of differentiation. In support of such "plasticity" in tissue macrophage responses, M1-type macrophages were shown to switch to an M2 phenotype depending on the composition of the local extracellular milieu (97). Furthermore, M1/M2 macrophage classification is largely based on in vitro differentiation, and increasing evidence suggests that such in vitro analyses do not accurately reflect the complexity of in vivo macrophage plasticity and heterogeneity. Adding to the complexity of macrophage classification, current evidence also suggests that monocytes that have entered tissues differentiate into macrophages displaying varying M1- or M2-like characteristics (98). These observations and emerging evidence increasingly imply that diverse macrophage populations mediate healing responses by releasing cytokines/chemokines, SPMs, proteases, and other mediators to orchestrate host defense, proliferation, and migration of wound-associated cells as well as matrix remodeling. Further studies are needed to better understand the role(s) of specific tissue macrophage subsets during the stages of wound repair (99).

Most tissues (including skin and intestine) contain macrophages termed tissue-resident macrophages (TRMs). TRMs represent a heterogeneous population of nonmigratory cells that respond to injury or infection by sensing DAMPs. TRMs in the skin and gut are continuously replenished by blood monocytes (100-102). TRM phenotypic markers vary depending on tissue

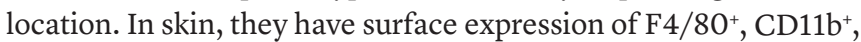
$\mathrm{CD}_{11} \mathrm{c}^{\mathrm{lo}}, \mathrm{CD}_{206}{ }^{+}, \mathrm{MHCII}^{\mathrm{lo}}$, Dectin- $1^{+}, \mathrm{CD} 01^{+}$, and Dectin- $2^{+}$, while in intestine, their cell surface markers include CX3CR-1 ${ }^{\text {hi }}$,

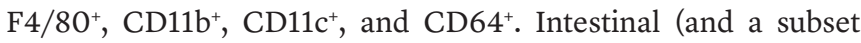
of dermal) $\mathrm{MHCII}^{\text {hi }}$ macrophages have been shown to originate from bone marrow monocytes. These macrophages lack capacity for self-renewal and have an estimated half-life of 4 to 6 weeks (103). An interesting subset of resident macrophages are epidermal Langerhans cells. While Langerhans cells were historically considered DCs, they are now believed to represent a specialized subset of TRMs. Unlike dermal TRMs, Langerhans cells are selfreplicating and can migrate to lymph nodes to present antigens to T cells (104). Importantly, Langerhans cells were reported to repopulate the epidermis during re-epithelialization of acute skin wounds (105). Despite these observations, mechanisms regulating TRM-mediated wound repair are poorly understood, although increasing reports implicate contributions of dermal TRMs to homeostatic maintenance, renewal of skin appendages, epithelial repair, and barrier recovery $(106,107)$. Important functions of intestinal TRMs include scavenging bacteria, helping maintain Tregs, and promoting epithelial cell renewal via production of IL-10 and prostaglandin $\mathrm{E}_{2}(89,100,108,109)$. Taken together, current evidence supports an emerging concept of multiple roles for TRMs, including maintenance of homeostasis in epithelial tis- sues as well as facilitating inflammatory responses and mediating repair following injury.

Macrophages in sites of injury are critical for skin and gut wound repair. Such wound-associated macrophages (WAMs) are adaptive, highly dynamic cells that can rapidly respond to cues within wound microenvironments (Figure 3). Current observations indicate that WAM phenotype is influenced by complex factors that are incompletely understood, including wound size, tissue location, and stage of the inflammatory process (acute versus chronic). Like other macrophages, WAMs can have varied M1/M2 phenotypes depending on the inflammatory/repair microenvironment, exhibiting characteristics of both proinflammatory and pro-repair macrophages (89). Given these observations, it is unsurprising that current literature is inconsistent on the types of cytokines produced by WAMs. Some studies report that WAMs are not an important source of the antiinflammatory cytokine IL-10 in skin wounds, whereas other groups demonstrate that both skin and gut WAMs are active producers of IL-10 with pro-repair properties $(110,111)$. Such discrepancies are likely related to the temporal nature of IL-10-dependent responses during wound repair. Supporting this notion, analyses of WAM-mediated IL-10 production suggest that IL-10 is produced at very specific times during the wound repair process, indicating that variables including wound size profoundly influence macrophage cytokine production responses.

Macrophages and other immune cells sense the metabolic environment and modulate function, an activity referred to as immunometabolism (112). Sites of injury have a hypoxic microenvironment generated primarily by neutrophils consuming high levels of oxygen while producing ROS in response to injury (113). Hypoxia also promotes increased HIF- $1 \alpha$ expression in inflammatory macrophages, which increases glycolytic enzyme expression and IL-1 $\beta$ synthesis (114). Balanced IL- $1 \beta$ release is important, as excess inflammasome signaling associated with IL-1 $\beta$ generation is linked to development of chronic wounds (115). Phagocytosis of cellular debris in association with IL-4 and IL-13 signaling facilitates dampening of inflammatory signals and initiation of the proliferative phase of tissue repair (116). Glucose is an important source of energy for inflammatory macrophage-mediated clearance of cellular debris that influences the proliferative phase in wound repair. Importantly, glucose availability likely influences macrophage secretion of proinflammatory mediators such as IL-1 $\beta$ and TNF- $\alpha$ (117). Interestingly, pro-repair macrophages have a highly oxidative metabolism, and therefore restoring oxygen levels is important in achieving resolution of inflammation (118).

Regenerative responses are likely mediated, in part, by intimate physical contact between macrophages and epithelial cells that promotes intestinal epithelial transcription of multiple prorepair genes, including Ccl-2, Cox-2, Igf-1, and Il-11 (Figure 3). Furthermore, since Cox-2 (encoding cyclooxygenase-2) is necessary for SPM synthesis, macrophage-mediated "activation" of epithelial cells might contribute to the generation of SPMs (119). Current evidence suggests that intestinal WAMs are required for amplification of colonic epithelial cell progenitors that contribute to wound repair. WAMs physically contact epithelial stem cells located within crypts, resulting in secretion of pro-proliferative and remodeling factors. Furthermore, recent evidence indicates that intestinal macrophages promote regenerative responses by 
integrating cues from mesenchymal stem cells, other immune cells, microbiota, and injured epithelia. Efficient colonic wound repair also depends on Trem 2 signaling in WAMs, which skews cellular machinery toward a pro-repair phenotype $(119,120)$. $\mathrm{CD}^{2} \mathrm{O6}^{+} \mathrm{CD}^{\mathrm{C}} 01 \mathrm{~b}^{+}$skin macrophages also produce the crucial pro-repair molecule TGF- $\beta 1$, a potent inducer of fibroblast proliferation and subsequent differentiation into myofibroblasts, leading to collagen deposition in the wound (121-125). WAMs also promote epithelial repair through release of IL-10 and PDGF- $\beta$ (125).

Macrophages can also directly transition into fibrosis-promoting cells, secreting ECM components such as collagen (126). These macrophages, referred to as fibrocytes or M2a macrophages, are implicated in pathogenesis of skin scarring. Interactions between macrophages and fibroblasts are critical in determining whether wounds heal with or without scarring. Regulatory-like, or M2c, macrophages within remodeling skin wounds release proteases and phagocytose cellular debris and ECM to clean out wounds and facilitate repair (127). In skin, WAMs are hypothesized to synthesize several members of the EGF family, e.g., EGF, TGF- $\alpha$, and heparin-bound EGF (EGF-HB), which enhance keratinocyte migration and proliferation, thereby promoting skin re-epithelialization (128-132). Inactive EGF family members are tethered to the cell membrane and require MMP-mediated cleavage to signal. Therefore, WAMs likely indirectly activate these growth factors by modulating MMP activity. IL-1, IL-6, TNF- $\alpha$, and TGF- $\beta$ also promote re-epithelialization (133). Interestingly, in human keratinocytes, WAM-derived TNF- $\alpha$ promotes expression of genes associated with cell movement, division, and survival (134). Presently, no studies highlight contributions of M2a and M2c macrophages to repair in the intestine. Recent observations indicate that WAMs in close proximity to wounded dermal and intestinal epithelial cells (and underlying fibroblasts) play important roles in orchestrating matrix remodeling and wound repair. As such, aberrations in macrophage function at different stages of wound repair markedly contribute to persistence of excessive ECM, resulting in skin fibrosis and permanent scarring.

Recently, further insights into the role of macrophages in wound repair have been gained from mouse models using depleted subsets of macrophages. Mice lacking the Spi-1 proto-oncogene protein lack mature macrophages as well as functional neutrophils. Surprisingly, these mice lack a skin wound-healing defect but rather exhibit marked reduction in scar formation (135). In support of macrophages' critical importance in skin wound repair, ablation of macrophages impaired murine skin wound healing (136, 137). These studies support an important role of macrophages in removing apoptotic neutrophils from wounds, thereby preventing ongoing release of tissue-degrading enzymes. Furthermore, when macrophages fail to appropriately clear apoptotic neutrophils, there are persistently high levels of proinflammatory cytokines and decreased local antiinflammatory and pro-repair mediators in wounds (138-140). Depletion of macrophages was also shown to reduce myofibroblast differentiation, which is necessary to promote wound contraction and accelerate skin wound healing (141).

Genetically engineered and inducible depletion models in mice enable selective macrophage depletion at different stages of the healing process, providing insights into the role of macrophages at various stages of wound repair. Macrophage depletion at early and mid-stages of skin repair results in delayed wound closure and decreased scar formation, while macrophage loss during later stages of repair did not affect healing. Depletion of macrophages at mid-stages of skin wound repair resulted in decreased VEGF-A and TGF- $\beta 1$ expression, as well as reduced angiogenesis and repair. Consistent with these observations, it was noted that during mid-stages of repair, macrophages secrete substantial amounts of VEGF-A and TGF- $\beta 1$ (106). The above-mentioned mouse models have not yet been used to study the role of macrophages in orchestrating intestinal mucosal repair in vivo. However, analogous temporal changes in macrophage function are likely necessary for mucosal repair in the gut. These findings highlight macrophages as critical to epithelial wound repair, displaying a dynamic capacity to polarize in response to environmental cues that change as wound healing progresses.

While we have discussed contribution of macrophages in orchestrating wound repair, DCs are also implicated as important innate mediators of repair. This topic is discussed in previous publications and reviews (142-144).

Therapeutic opportunities. Several studies have either targeted neutrophils/macrophages or used these cells as tools as part of strategies to improve wound healing. Nevertheless, such therapeutic targeting of innate immune cells has been limited by incomplete understanding of underlying mechanisms by which these cell populations regulate repair. Early research focusing on promoting neutrophil apoptosis yielded promising results, but off-target cell death presented a challenge (145). Novel technologies and drugs aided in the development of new strategies to promote wound repair by inducing resolution of inflammation without reducing neutrophil recruitment. A recent study observed that neutrophils "retrotax," or reverse-migrate, away from inflammatory sites when exposed to SPMs (146). Manipulating this process could potentially improve healing as well as infection control. Other studies showed potential "therapeutic benefit" through controlled delivery of leukocyte-derived SPMs. For example, nanoparticles containing neutrophil-derived microparticles with aspirin-triggered resolvin D1 or lipoxin $\mathrm{A}_{4}$ analogs reduced neutrophil recruitment in murine peritonitis and accelerated keratinocyte wound healing (147).

Strategies to improve wound healing through increased macrophage recruitment and polarization toward a pro-repair phenotype have also been investigated. Direct injection of IL-1 $\beta$-activated macrophages into murine skin wounds increased VEGF-C production and improved wound repair (148). Furthermore, local GM-CSF application to dermal wounds resulted in increased WAMs and enhanced wound healing (149). Since the complex biomolecular microenvironment within wounds plays a critical role in regulating macrophage polarization, strategies to enhance production or delivery of pro-repair molecules have been explored. For example, glutamine-loaded hydrogels increased the rate of wound closure and re-epithelialization in wounded skin. In this study, collagen deposition within wounds was consistent with increased activity of alternatively activated macrophages (150). Conversely, strategies targeting inhibition of alternative macrophage activation and resulting Arg-1 activity may help prevent scarring and fibrosis by reducing excessive collagen deposition (151). From these observations, it is clear that methods promot- 
ing macrophage activity or polarization to a wound-healing phenotype have considerable promise, further supported by multiple other reports employing mesenchymal stem cells, growth factors, and biomaterials to modulate macrophage phenotype, function, and transcriptome (152-154).

\section{Concluding remarks}

Repair of injured epithelial barriers is a highly regulated process orchestrated by resident cells and spatiotemporal immune cell recruitment, which not only contributes to host defense but is vital for tissue homeostasis and wound repair. Temporal interplay between immune cells and wound-associated cells, secreted proteins, and lipids ensures efficient resolution of inflammation in concert with epithelial repair. One caveat is that most wound-healing research is performed in animal models, raising the question of relevance to human health. Notably, the relative abundance of circulating neutrophils and monocytes in the blood differs considerably between humans (50\%-70\% neutrophils, 10\% monocytes) and mice (10\%-25\% neutrophils, $4 \%$ monocytes). However, many studies report similar dynamics of innate immune cell recruitment to sites of injury in mice and humans. Furthermore, a similar prevalence of activated neutrophils is observed in chronic nonhealing wounds of both species, highlighting the relevance of murine models for studies of innate immune cell biology in wound healing (155157). Human and mouse mononuclear phagocytes lack overlapping phenotypic markers, a challenge that hinders the identification and characterization of homologous populations between species
(158). To overcome challenges arising from species differences, new approaches using transcriptomics, metabolomics, humanized mice, and simple human/animal models (such as the skin blister model) must be exploited to directly compare and contrast functional biology of immune cell subsets between species (159-161).

While this brief overview highlights increased mechanistic evidence of the role of epithelial cells, neutrophils, monocytes, and macrophages in orchestrating skin and intestinal wound repair, it is also clear that many other cellular contributions remain understudied. Given the plethora of chronic diseases associated with impaired wound-healing responses, much investigation remains to facilitate design of new therapeutic approaches to promote repair of wounds in chronic diseases.

\section{Acknowledgments}

This work was supported by NIH grants (DK055679, DK089763, and DK059888 to AN; and DK61739, DK72564, and DK79392 to CAP) and a Crohn's and Colitis Foundation Senior Research Award (544596 to JCB) and Career Development Award (544599 to MQ).

Address correspondence to: Charles A. Parkos, Department of Pathology, University of Michigan Medical School, 4063 BSRB, 109 Zina Pitcher Place, Ann Arbor, Michigan 48109-2200, USA. Phone: 734.763.6384; Email: cparkos@umich.edu. Or to: Asma Nusrat, Department of Pathology, University of Michigan Medical School, 4057 BSRB, 109 Zina Pitcher Place, Ann Arbor, Michigan 481092200, USA. Phone: 734.764.5712; Email: anusrat@umich.edu.
1. Dahlhamer JM, Zammitti EP, Ward BW, Wheaton AG, Croft JB. Prevalence of inflammatory bowel disease among adults aged $\geq 18$ years - United States, 2015. MMWR Morb Mortal Wkly Rep. 2016;65(42):1166-1169.

2. Kappelman MD, et al. Direct health care costs of Crohn's disease and ulcerative colitis in US children and adults. Gastroenterology. 2008;135(6):1907-1913.

3. Baumgart DC, Sandborn WJ. Inflammatory bowel disease: clinical aspects and established and evolving therapies. Lancet. 2007;369(9573):1641-1657.

4. Rieder F, Brenmoehl J, Leeb S, Schölmerich J, Rogler G. Wound healing and fibrosis in intestinal disease. Gut. 2007;56(1):130-139.

5. Sen CK, et al. Human skin wounds: a major and snowballing threat to public health and the economy. Wound Repair Regen. 2009;17(6):763-771.

6. Sturm A, Dignass AU. Epithelial restitution and wound healing in inflammatory bowel disease. World J Gastroenterol. 2008;14(3):348-353.

7. McCormack SA, Viar MJ, Johnson LR. Migration of IEC-6 cells: a model for mucosal healing. Am J Physiol. 1992;263(3 pt 1):G426-G435.

8. Nusrat A, Delp C, Madara JL. Intestinal epithelial restitution. Characterization of a cell culture model and mapping of cytoskeletal elements in migrating cells. JClin Invest. 1992;89(5):1501-1511.

9. Moore R, Carlson S, Madara JL. Rapid barrier restitution in an in vitro model of intestinal epithelial injury. Lab Invest. 1989;60(2):237-244.

10. Feil W, Wenzl E, Vattay P, Starlinger M, Sogukoglu T, Schiessel R. Repair of rabbit duodenal mucosa after acid injury in vivo and in vitro. Gas troenterology. 1987;92(6):1973-1986.

11. Leoni G, Neumann PA, Sumagin R, Denning TL, Nusrat A. Wound repair: role of immuneepithelial interactions. Mucosal Immunol. 2015;8(5):959-968.

12. Lotz MM, Nusrat A, Madara JL, Ezzell R, Wewer UM, Mercurio AM. Intestinal epithelial restitution. Involvement of specific laminin isoforms and integrin laminin receptors in wound closure of a transformed model epithelium. Am J Pathol. 1997;150(2):747-760.

13. van der Flier LG, Clevers H. Stem cells, selfrenewal, and differentiation in the intestinal epithelium. Annu Rev Physiol. 2009;71:241-260.

14. Peterson LW, Artis D. Intestinal epithelial cells: regulators of barrier function and immune homeostasis. Nat Rev Immunol. 2014;14(3):141-153.

15. Burclaff J, Mills JC. Plasticity of differentiated cells in wound repair and tumorigenesis, part II: skin and intestine. Dis Model Mech. 2018;11(9):dmm035071.

16. Aragona $\mathrm{M}$, et al. Defining stem cell dynamics and migration during wound healing in mouse skin epidermis. Nat Commun. 2017;8:14684.

17. Sun BK, Siprashvili Z, Khavari PA. Advances in skin grafting and treatment of cutaneous wounds. Science. 2014;346(6212):941-945.

18. Gurtner GC, Werner S, Barrandon Y, Longaker MT. Wound repair and regeneration. Nature. 2008;453(7193):314-321.

19. Caley MP, Martins VL, O'Toole EA. Metalloproteinases and wound healing. Adv Wound Care (New Rochelle). 2015;4(4):225-234.
20. Rohani MG, Parks WC. Matrix remodeling by MMPs during wound repair. Matrix Biol. 2015;44-46:113-121.

21. Li Y, Kilani RT, Rahmani-Neishaboor E, Jalili RB, Ghahary A. Kynurenine increases matrix metalloproteinase- 1 and -3 expression in cultured dermal fibroblasts and improves scarring in vivo. J Invest Dermatol. 2014;134(3):643-650.

22. Eto H, et al. Therapeutic potential of fibroblast growth factor-2 for hypertrophic scars: upregulation of MMP-1 and HGF expression. Lab Invest. 2012;92(2):214-223.

23. Matsuno K, et al. The expression of matrix metalloproteinase matrilysin indicates the degree of inflammation in ulcerative colitis. J Gastroenterol. 2003;38(4):348-354.

24. Saarialho-Kere UK, Vaalamo M, Puolakkainen $P$ Airola K, Parks WC, Karjalainen-Lindsberg ML. Enhanced expression of matrilysin, collagenase, and stromelysin-1 in gastrointestinal ulcers. Am J Pathol. 1996;148(2):519-526.

25. Enyedi B, Niethammer P. Mechanisms of epithelial wound detection. Trends Cell Biol. 2015;25(7):398-407.

26. Yamaguchi N, Mizutani T, Kawabata K, Haga H. Leader cells regulate collective cell migration via Rac activation in the downstream signaling of integrin $\beta 1$ and PI3K. Sci Rep. 2015;5:7656.

27. Moyer RA, Wendt MK, Johanesen PA, Turner JR, Dwinell MB. Rho activation regulates CXCL12 chemokine stimulated actin rearrangement and restitution in model intestinal epithelia. $L a b$ Invest. 2007;87(8):807-817.

28. Babbin BA, et al. Annexin 2 regulates intestinal 
epithelial cell spreading and wound closure through Rho-related signaling. Am J Pathol. 2007;170(3):951-966.

29. Hinrichs $B H$, et al. Serum smyloid $A 1$ is an epithelial prorestitutive factor. Am J Pathol. 2018;188(4):937-949.

30. Leoni G, et al. Annexin A1, formyl peptide receptor, and NOX1 orchestrate epithelial repair. JClin Invest. 2013;123(1):443-454.

31. Miyoshi H, Ajima R, Luo CT, Yamaguchi TP, Stappenbeck TS. Wnt5a potentiates TGF- $\beta$ signaling to promote colonic crypt regeneration after tissue injury. Science. 2012;338(6103):108-113.

32. Bradford EM, et al. Epithelial TNF receptor signaling promotes mucosal repair in inflammatory bowel disease. J Immunol. 2017;199(5):1886-1897.

33. Birkl D, et al. TNF $\alpha$ promotes mucosal wound repair through enhanced platelet activating factor receptor signaling in the epithelium. Mucosal Immunol. 2019;12(4):909-918.

34. Kim MH, et al. Dynamics of neutrophil infiltration during cutaneous wound healing and infection using fluorescence imaging. J Invest Dermatol. 2008;128(7):1812-1820.

35. Kolaczkowska E, Kubes P. Neutrophil recruitment and function in health and inflammation. Nat Rev Immunol. 2013;13(3):159-175.

36. Williams IR, Parkos CA. Colonic neutrophils in inflammatory bowel disease: double-edged swords of the innate immune system with protective and destructive capacity. Gastroenterology. 2007;133(6):2049-2052.

37. Anderson DC, et al. The severe and moderate phenotypes of heritable Mac-1, LFA-1 deficiency: their quantitative definition and relation to leukocyte dysfunction and clinical features. J Infect Dis. 1985;152(4):668-689.

38. Bressenot A, Salleron J, Bastien C, Danese S, Boulagnon-Rombi C, Peyrin-Biroulet L. Comparing histological activity indexes in UC. Gut. 2015;64(9):1412-1418.

39. Riley SA, Mani V, Goodman MJ, Dutt S, Herd ME. Microscopic activity in ulcerative colitis: what does it mean? Gut.1991;32(2):174-178.

40. Geboes K, Riddell R, Ost A, Jensfelt B, Persson T, Löfberg R. A reproducible grading scale for histological assessment of inflammation in ulcerative colitis. Gut. 2000;47(3):404-409.

41. Mori R, Kondo T, Nishie T, Ohshima T, Asano M. Impairment of skin wound healing in $\beta$-1,4-galactosyltransferase-deficient mice with reduced leukocyte recruitment. Am J Pathol. 2004;164(4):1303-1314.

42. Devalaraja RM, et al. Delayed wound healing in CXCR2 knockout mice. J Invest Dermatol. 2000;115(2):234-244.

43. Kuhl AA, et al. Aggravation of different types of experimental colitis by depletion or adhesion blockade of neutrophils. Gastroenterology. 2007;133(6):1882-1892.

44. Zhang R, Ito S, Nishio N, Cheng Z, Suzuki H, Isobe K. Up-regulation of $\mathrm{Gr}^{+} \mathrm{CD} 11 \mathrm{~b}^{+}$population in spleen of dextran sulfate sodium administered mice works to repair colitis. Inflamm Allergy Drug Targets. 2011;10(1):39-46.

45. Lekstrom-Himes JA, Gallin JI. Immunodeficiency diseases caused by defects in phagocytes. $\mathrm{N} \mathrm{Engl}$ JMed. 2000;343(23):1703-1714.
46. Nathan C. Neutrophils and immunity: challenges and opportunities. Nat Rev Immunol. 2006;6(3):173-182.

47. Ley K, Laudanna C, Cybulsky MI, Nourshargh S. Getting to the site of inflammation: the leukocyte adhesion cascade updated. Nat Rev Immunol. 2007;7(9):678-689.

48. Brazil JC, Parkos CA. Pathobiology of neutrophil-epithelial interactions. Immunol Rev. 2016;273(1):94-111.

49. Brinkmann V, et al. Neutrophil extracellular traps kill bacteria. Science. 2004;303(5663):1532-1535.

50. MacLeod AS, Mansbridge JN. The innate immune system in acute and chronic wounds. Adv Wound Care (New Rochelle). 2016;5(2):65-78.

51. Kominsky DJ, Campbell EL, Colgan SP. Metabolic shifts in immunity and inflammation. J Immunol. 2010;184(8):4062-4068.

52. Campbell EL, et al. Transmigrating neutrophils shape the mucosal microenvironment through localized oxygen depletion to influence resolution of inflammation. Immunity. 2014;40(1):66-77.

53. Furuta GT, et al. Hypoxia-inducible factor 1-dependent induction of intestinal trefoil factor protects barrier function during hypoxia. J Exp Med.2001;193(9):1027-1034.

54. Hong WX, et al. The role of hypoxia-inducible factor in wound healing. Adv Wound Care (New Rochelle). 2014;3(5):390-399.

55 . Botusan IR, et al. Stabilization of HIF-1 $\alpha$ is critica to improve wound healing in diabetic mice. Proc Natl Acad Sci U S A. 2008;105(49):19426-19431.

56 . Chen F, et al. Neutrophils promote amphiregulin production in intestinal epithelial cells through TGF- $\beta$ and contribute to intestinal homeostasis. JImmunol. 2018;201(8):2492-2501.

57. Zaiss DMW, Gause WC, Osborne LC, Artis D. Emerging functions of amphiregulin in orchestrating immunity, inflammation, and tissue repair. Immunity. 2015;42(2):216-226.

58. Ramirez H, Patel SB, Pastar I. The role of TGF $\beta$ signaling in wound epithelialization. Adv Wound Care (New Rochelle). 2014;3(7):482-491.

59. Buschke S, et al. A decisive function of transforming growth factor- $\beta /$ Smad signaling in tissue morphogenesis and differentiation of human $\mathrm{HaCaT}$ keratinocytes. Mol Biol Cell. 2011;22(6):782-794.

60. Cox DA, Kunz S, Cerletti N, McMaster GK, Burk RR. Wound healing in aged animals - effects of locally applied transforming growth factor beta 2 in different model systems. In: Steiner R, Weisz PB, Langer R, eds. Angiogenesis. Basel, Switzerland; Birkhäuser: 1992;61:287-295.

61. Gillitzer R, Goebeler M. Chemokines in cutaneous wound healing. J Leukoc Biol. 2001;69(4):513-521.

62. Theilgaard-Mönch K, Knudsen S, Follin P, Borregaard $\mathrm{N}$. The transcriptional activation program of human neutrophils in skin lesions supports their important role in wound healing. J Immunol. 2004;172(12):7684-7693.

63. Sørensen OE, Cowland JB, Theilgaard-Mönch K, Liu L, Ganz T, Borregaard N. Wound healing and expression of antimicrobial peptides/ polypeptides in human keratinocytes, a consequence of common growth factors. J Immunol. 2003;170(11):5583-5589.

64. Feiken E, Rømer J, Eriksen J, Lund LR. Neutrophils express tumor necrosis factor-alpha during mouse skin wound healing. J Invest Dermatol. 1995;105(1):120-123.

65. Kanno E, et al. Wound healing in skin promoted by inoculation with Pseudomonas aeruginosa PAO1: the critical role of tumor necrosis factor- $\alpha$ secreted from infiltrating neutrophils. Wound Repair Regen. 2011;19(5):608-621.

66. Frank JB, et al. In vivo effect of tumor necrosis factor $\alpha$ on wound angiogenesis and epithelization. Eur J Trauma. 2003;29(4):208-219.

67. Scapini P, et al. Neutrophils produce biologically active macrophage inflammatory protein-30


Immunol. 2001;31(7):1981-1988.

68. Le Borgne M, et al. Dendritic cells rapidly recruited into epithelial tissues via CCR6/CCL20 are responsible for $\mathrm{CD} 8^{+} \mathrm{T}$ cell crosspriming in vivo. Immunity. 2006;24(2):191-201.

69. Kvedaraite E, et al. Tissue-infiltrating neutrophils represent the main source of IL-23 in the colon of patients with IBD. Gut. 2016;65(10):1632-1641.

70. Zindl CL, et al. IL-22-producing neutrophils contribute to antimicrobial defense and restitution of colonic epithelial integrity during colitis. Proc Natl Acad Sci U S A. 2013;110(31):12768-12773.

71. Zhou G, et al. CD177 neutrophils as functionally activated neutrophils negatively regulate IBD. Gut. 2018;67(6):1052-1063.

72. Denning TL, Parkos CA. Neutrophils enlist IL-22 to restore order in the gut. Proc Natl Acad Sci U S A. 2013;110(31):12509-12510.

73. Medina-Contreras O, et al. Cutting edge: IL-36 receptor promotes resolution of intestinal damage. J Immunol. 2016;196(1):34-38.

74. Dumoutier L, Lejeune D, Hor S, Fickenscher H, Renauld JC. Cloning of a new type II cytokine receptor activating signal transducer and activator of transcription (STAT)1, STAT2 and STAT3. Biochem J. 2003;370(pt 2):391-396.

75. McGee HM, et al. IL-22 promotes fibroblastmediated wound repair in the skin. J Invest Dermatol. 2013;133(5):1321-1329.

76. Curtis VF, et al. Neutrophils as sources of dinucleotide polyphosphates and metabolism by epithelial ENPP1 to influence barrier function via adenosine signaling. Mol Biol Cell. 2018;29(22):2687-2699.

77. Colgan SP, Eltzschig HK. Adenosine and hypoxiainducible factor signaling in intestinal injury and recovery. Annu Rev Physiol. 2012;74:153-175.

78. Colgan SP. Neutrophils and inflammatory resolution in the mucosa. Semin Immunol. 2015;27(3):177-183.

79. Ivanov AI, Parkos CA, Nusrat A. Cytoskeletal regulation of epithelial barrier function during inflammation. Am J Pathol. 2010;177(2):512-524.

80. Montesinos MC, et al. Adenosine promotes wound healing and mediates angiogenesis in response to tissue injury via occupancy of $\mathrm{A}(2 \mathrm{~A})$ receptors. Am J Pathol. 2002;160(6):2009-2018.

81. Wang J, Hossain M, Thanabalasuriar A, Gunzer $\mathrm{M}$, Meininger C, Kubes P. Visualizing the function and fate of neutrophils in sterile injury and repair. Science. 2017;358(6359):111-116.

82. Hasenberg A, et al. Catchup: a mouse model for imaging-based tracking and modulation of neutrophil granulocytes. Nat Methods. 2015;12(5):445-452. 
83. Fridlender ZG, et al. Polarization of tumor-associated neutrophil phenotype by TGF- $\beta$ : "N1" versus “N2" TAN. Cancer Cell. 2009;16(3):183-194.

84. Deshmane SL, Kremlev S, Amini S, Sawaya BE. Monocyte chemoattractant protein-1 (MCP-1): an overview. J Interferon Cytokine Res. 2009;29(6):313-326.

85. Martins-Green M, Petreaca M, Wang L. Chemokines and their receptors are key players in the orchestra that regulates wound healing. $A d v$ Wound Care (New Rochelle). 2013;2(7):327-347.

86. Ishida Y, Gao JL, Murphy PM. Chemokine receptor CX3CR1 mediates skin wound healing by promoting macrophage and fibroblast accumulation and function. JImmunol. 2008;180(1):569-579.

87. Zigmond $\mathrm{E}$, et al. Ly6C hi monocytes in the inflamed colon give rise to proinflammatory effector cells and migratory antigen-presenting cells. Immunity. 2012;37(6):1076-1090.

88. Shi C, Pamer EG. Monocyte recruitment during infection and inflammation. Nat Rev Immunol. 2011;11(11):762-774.

89. Brancato SK, Albina JE. Wound macrophages as key regulators of repair: origin, phenotype, and function. Am J Pathol. 2011;178(1):19-25.

90. Tamoutounour S, et al. Origins and functional specialization of macrophages and of conventional and monocyte-derived dendritic cells in mouse skin. Immunity. 2013;39(5):925-938.

91. Ginhoux F, Jung S. Monocytes and macrophages: developmental pathways and tissue homeostasis. Nat Rev Immunol. 2014;14(6):392-404.

92. Elliott MR, Koster KM, Murphy PS. Efferocytosis signaling in the regulation of macrophage inflammatory responses. J Immunol. 2017;198(4):1387-1394.

93. Kloc M, Ghobrial RM, Wosik J, Lewicka A, Lewicki S, Kubiak JZ. Macrophage functions in wound healing. J Tissue Eng Regen Med. 2019;13(1):99-109.

94. Quiros M, Nusrat A. Saving problematic mucosae: SPMs in intestinal mucosal inflammation and repair. Trends Mol Med. 2019;25(2):124-135.

95. Mantovani A, Sica A, Locati M. Macrophage polarization comes of age. Immunity. 2005;23(4):344-346.

96. Dalli J, Serhan C. Macrophage proresolving mediators - the when and where. Microbiol Spectr. 2016;4(3):MCHD-0001-2014.

97. Cohen HB, Mosser DM. Extrinsic and intrinsic control of macrophage inflammatory responses. J Leukoc Biol. 2013;94(5):913-919.

98. Guilliams M, Mildner A, Yona S. Developmental and functional heterogeneity of monocytes. Immunity. 2018;49(4):595-613.

99. Wynn TA, Vannella KM. Macrophages in tissue repair, regeneration, and fibrosis. Immunity. 2016;44(3):450-462.

100.Shaw TN, et al. Tissue-resident macrophages in the intestine are long lived and defined by Tim- 4 and CD4 expression. J Exp Med. 2018;215(6):1507-1518.

101. Bain CC, et al. Constant replenishment from circulating monocytes maintains the macrophage pool in the intestine of adult mice. Nat Immunol. 2014;15(10):929-937.

102.Wang Y, et al. IL-34 is a tissue-restricted ligand of CSF1R required for the development of Langerhans cells and microglia. Nat Immunol.
2012;13(8):753-760.

103. Sheng J, Ruedl C, Karjalainen K. Most tissueresident macrophages except microglia are derived from fetal hematopoietic stem cells. Immunity. 2015;43(2):382-393.

104.Doebel T, Voisin B, Nagao K. Langerhans cells the macrophage in dendritic cell clothing. Trends Immunol. 2017;38(11):817-828.

105. Stojadinovic O, Yin N, Lehmann J, Pastar I, Kirsner RS, Tomic-Canic M. Increased number of Langerhans cells in the epidermis of diabetic foot ulcers correlates with healing outcome. Immunol Res. 2013;57(1-3):222-228.

106.Lucas T, et al. Differential roles of macrophages in diverse phases of skin repair. JImmunol. 2010;184(7):3964-3977.

107. Davies LC, Jenkins SJ, Allen JE, Taylor PR. Tissue-resident macrophages. Nat Immunol. 2013;14(10):986-995.

108. Dejani NN, et al. Intestinal host defense outcome is dictated by PGE. Proc Natl Acad Sci US A. 2018;115(36):E8469-E8478.

109. Denning TL, Wang YC, Patel SR, Williams IR, Pulendran B. Lamina propria macrophages and dendritic cells differentially induce regulatory and interleukin 17-producing $\mathrm{T}$ cell responses. Nat Immunol. 2007;8(10):1086-1094.

110.Daley JM, Brancato SK, Thomay AA, Reichner JS, Albina JE. The phenotype of murine wound macrophages. J Leukoc Biol. 2010;87(1):59-67.

111. Quiros M, et al. Macrophage-derived IL-10 mediates mucosal repair by epithelial WISP-1 signaling. JClin Invest. 2017;127(9):3510-3520.

112. O’Neill LA, Kishton RJ, Rathmell J. A guide to immunometabolism for immunologists. Nat Rev Immunol. 2016;16(9):553-565.

113. de Oliveira S, Rosowski EE, Huttenlocher A. Neutrophil migration in infection and wound repair: going forward in reverse. Nat Rev Immunol. 2016;16(6):378-391.

114. Tannahill GM, et al. Succinate is an inflammatory signal that induces IL- $1 \beta$ through HIF- $1 \alpha$. Nature. 2013;496(7444):238-242.

115. Mirza RE, Fang MM, Ennis WJ, Koh TJ. Blocking interleukin- $1 \beta$ induces a healing-associated wound macrophage phenotype and improves healing in type 2 diabetes. Diabetes. 2013;62(7):2579-2587.

116. Bosurgi L, et al. Macrophage function in tissue repair and remodeling requires IL- 4 or IL-13 with apoptotic cells. Science. 2017;356(6342):1072-1076.

117. Freemerman AJ, et al. Metabolic reprogramming of macrophages: glucose transporter 1 (GLUT1)-mediated glucose metabolism drives a proinflammatory phenotype. J Biol Chem 2014;289(11):7884-7896.

118. Vats D, et al. Oxidative metabolism and PGC-1 $\beta$ attenuate macrophage-mediated inflammation. Cell Metab. 2006;4(1):13-24.

119. Seno H, Miyoshi H, Brown SL, Geske MJ, Colonna M, Stappenbeck TS. Efficient colonic mucosal wound repair requires Trem 2 signaling. Proc Natl Acad Sci U S A . 2009;106(1):256-261.

120. Pull SL, Doherty JM, Mills JC, Gordon JI, Stappenbeck TS. Activated macrophages are an adaptive element of the colonic epithelial progenitor niche necessary for regenerative responses to injury. Proc Natl Acad Sci U S A. 2005;102(1):99-104
121. Desmoulière A, Geinoz A, Gabbiani F, Gabbiani $\mathrm{G}$. Transforming growth factor- $\beta 1$ induces $\alpha$-smooth muscle actin expression in granulation tissue myofibroblasts and in quiescent and growing cultured fibroblasts. J Cell Biol. 1993;122(1):103-111.

122. Pakyari M, Farrokhi A, Maharlooei MK, Ghahary A. Critical role of transforming growth factor $\beta$ in different phases of wound healing. Adv Wound Care (New Rochelle). 2013;2(5):215-224.

123. Nacu N, et al. Macrophages produce TGF$\beta$-induced ( $\beta$-ig-h3) following ingestion of apoptotic cells and regulate MMP14 levels and collagen turnover in fibroblasts. JImmunol. 2008;180(7):5036-5044.

124. Liu Y, et al. TGF- $\beta 1$ promotes scar fibroblasts proliferation and transdifferentiation via up-regulating MicroRNA-21. Sci Rep. 2016;6:32231.

125. Shook B, Xiao E, Kumamoto Y, Iwasaki A, Horsley V. CD301 $\mathrm{b}^{+}$macrophages are essential for effective skin wound healing. J Invest Dermatol. 2016;136(9):1885-1891.

126.Schnoor M, et al. Production of type VI collagen by human macrophages: a new dimension in macrophage functional heterogeneity. J Immunol. 2008;180(8):5707-5719.

127. Hesketh M, Sahin KB, West ZE, Murray RZ. Macrophage phenotypes regulate scar formation and chronic wound healing. Int J Mol Sci. 2017;18(7):E1545.

128. Rheinwald JG, Green H. Epidermal growth factor and the multiplication of cultured human epidermal keratinocytes. Nature. 1977;265(5593):421-424.

129.Li Y, Fan J, Chen M, Li W, Woodley DT. Transforming growth factor-alpha: a major human serum factor that promotes human keratinocyte migration. J Invest Dermatol. 2006;126(9):2096-2105.

130. Schultz G, Rotatori DS, Clark W. EGF and TGFin wound healing and repair.J Cell Biochem. 1991;45(4):346-352.

131. Marikovsky M, et al. Wound fluid-derived heparin-binding EGF-like growth factor (HB-EGF) is synergistic with insulin-like growth factor-I for Balb/MK keratinocyte proliferation. J Invest Dermatol.1996;106(4):616-621.

132. Shirakata Y, et al. Heparin-binding EGF-like growth factor accelerates keratinocyte migration and skin wound healing. J Cell Sci. 2005;118(pt 11):2363-2370.

133. Martinez FO, Helming L, Gordon S. Alternative activation of macrophages: an immunologic functional perspective. Annu Rev Immunol. 2009;27:451-483.

134. Banno T, Gazel A, Blumenberg M. Effects of tumor necrosis factor- $\alpha$ (TNF- $\alpha)$ in epidermal keratinocytes revealed using global transcriptional profiling. J Biol Chem. 2004;279(31):32633-32642.

135. Martin P, et al. Wound healing in the PU.1 null mouse - tissue repair is not dependent on inflammatory cells. Curr Biol. 2003;13(13):1122-1128.

136. Goren I, et al. A transgenic mouse model of inducible macrophage depletion: effects of diphtheria toxin-driven lysozyme M-specific cell lineage ablation on wound inflammatory, angiogenic, and contractive processes. Am J Pathol. 2009;175(1):132-147. 
137. Mirza R, DiPietro LA, Koh TJ. Selective and specific macrophage ablation is detrimental to wound healing in mice. Am J Pathol. 2009;175(6):2454-2462.

138. Daley JM, et al. Modulation of macrophage phenotype by soluble product(s) released from neutrophils. J Immunol. 2005;174(4):2265-2272.

139. Meszaros AJ, Reichner JS, Albina JE. Macrophage-induced neutrophil apoptosis. J Immunol. 2000;165(1):435-441.

140. Meszaros AJ, Reichner JS, Albina JE. Macrophage phagocytosis of wound neutrophils. J Leukoc Biol. 1999;65(1):35-42.

141. Bochaton-Piallat ML, Gabbiani G, Hinz B. The myofibroblast in wound healing and fibrosis: answered and unanswered questions. F1000Res. 2016;5:F1000 Faculty Rev-752.

142.Strbo N, Yin N, Stojadinovic O. Innate and adaptive immune responses in wound epithelialization. Adv Wound Care (New Rochelle). 2014;3(7):492-501.

143. Haniffa M, Gunawan M, Jardine L. Human skin dendritic cells in health and disease. J Dermatol Sci. 2015;77(2):85-92.

144. Kadowaki T, Shimada M, Inagawa H, Kohchi C, Hirashima M, Soma G. Reconsideration of macrophage and dendritic cell classification. Anticancer Res. 2012;32(6):2257-2261.

145. Rossi AG, et al. Cyclin-dependent kinase inhibitors enhance the resolution of inflammation by promoting inflammatory cell apoptosis. Nat Med. 2006;12(9):1056-1064.
146. Robertson AL, et al. A zebrafish compound screen reveals modulation of neutrophil reverse migration as an anti-inflammatory mechanism. Sci Transl Med. 2014;6(225):225ra29.

147. Norling LV, Spite M, Yang R, Flower RJ, Perretti M, Serhan CN. Cutting edge: humanized nano-proresolving medicines mimic inflammation-resolution and enhance wound healing. JImmunol. 2011;186(10):5543-5547.

148. Maruyama K, Asai J, Ii M, Thorne T, Losordo DW, D'Amore PA. Decreased macrophage number and activation lead to reduced lymphatic vessel formation and contribute to impaired diabetic wound healing. Am J Pathol. 2007;170(4):1178-1191.

149. Fang Y, Shen J, Yao M, Beagley KW, Hambly BD, Bao S. Granulocyte-macrophage colony-stimulating factor enhances wound healing in diabetes via upregulation of proinflammatory cytokines. Br J Dermatol. 2010;162(3):478-486.

150. Thangavel P, Ramachandran B, Chakraborty S, Kannan R, Lonchin S, Muthuvijayan V. Accelerated healing of diabetic wounds treated with l-glutamic acid loaded hydrogels through enhanced collagen deposition and angiogenesis: an in vivo study. Sci Rep. 2017;7(1):10701.

151. Campbell L, Saville CR, Murray PJ, Cruickshank SM, Hardman MJ. Local arginase 1 activity is required for cutaneous wound healing. JInvest Dermatol. 2013;133(10):2461-2470.

152. Hu MS, et al. Delivery of monocyte lineage cells in a biomimetic scaffold enhances tissue repair. JCI Insight. 2017;2(19):96260.
153. Barminko JA, Nativ NI, Schloss R, Yarmush ML. Fractional factorial design to investigate stromal cell regulation of macrophage plasticity. Biotechnol Bioeng. 2014;111(11):2239-2251.

154. Self-Fordham JB, Naqvi AR, Uttamani JR, Kulkarni V, Nares S. MicroRNA: dynamic regulators of macrophage polarization and plasticity. Front Immunol. 2017;8:1062.

155. Doeing DC, Borowicz JL, Crockett ET. Gender dimorphism in differential peripheral blood leukocyte counts in mice using cardiac, tail, foot, and saphenous vein puncture methods. BMC Clin Pathol. 2003;3(1):3.

156. Mestas J, Hughes CC. Of mice and not men: differences between mouse and human immunology. JImmunol. 2004;172(5):2731-2738.

157. van Furth R, Sluiter W, van Dissel JT. Genetic control of macrophage responses. Ann N Y Acad Sci. 1986;465:15-25.

158. Reynolds G, Haniffa M. Human and mouse mononuclear phagocyte networks: a tale of two species? Front Immunol. 2015;6:330.

159. van de Mortel JE, Aarts MG. Comparative transcriptomics - model species lead the way. New Phytol. 2006;170(2):199-201.

160. Shay T, et al. Conservation and divergence in the transcriptional programs of the human and mouse immune systems. Proc Natl Acad Sci US A. 2013;110(8):2946-2951.

161. Jenner W, et al. Characterisation of leukocytes in a human skin blister model of acute inflammation and resolution. PLoS One. 2014;9(3):e89375. 